\title{
Existence of heteroclinic solution for a class of non-autonomous second-order equation
}

\author{
Claudianor O. Alves
}

\begin{abstract}
In this paper, we use variational methods to prove the existence of heteroclinic solutions for a class of non-autonomous second-order equation.
\end{abstract}

Mathematics Subject Classification. 34C37, 37J45, 46E35.

Keywords. Heteroclinic solutions, Minimization, Second-order equation.

\section{Introduction}

Consider the non-autonomous second-order differential equation

$$
\begin{gathered}
\ddot{x}(t)=a(\epsilon t) V^{\prime}(x(t)), \quad t \in \mathbb{R}, \\
x(t) \rightarrow-1 \text { as } t \rightarrow-\infty, \quad x(t) \rightarrow 1 \text { as } t \rightarrow+\infty,
\end{gathered}
$$

where $\epsilon>0$ is a positive parameter and $V: \mathbb{R} \rightarrow \mathbb{R}$ is a function verifying:

$\left(V_{1}\right) \quad V \in C^{2}\left(\mathbb{R}^{N}, \mathbb{R}\right)$.

$\left(V_{2}\right) \quad V(t) \geq 0 \forall t \in \mathbb{R}$ and $V(-1)=V(1)=0$.

$\left(V_{3}\right) \quad V(t)>0$ for all $t \in(-1,1)$,

and

$\left(V_{4}\right) \quad V^{\prime \prime}(-1), V^{\prime \prime}(1)>0$.

Related to function $a: \mathbb{R} \rightarrow \mathbb{R}$, we assume that it is a bounded continuous function satisfying some conditions which will be mentioned later on.

The main goal of the present paper is to prove the existence of solution for problem (1.1)-(1.2), which is called a heteroclinic solution, connecting the equilibria -1 and 1 .

The existence of heteroclinic solution has received a special attention, because this type of solution appears in a lot of mathematical models, such

Research of C. O. Alves partially supported by CNPq 304036/2013-7 and INCT-MAT. 
as Mechanics, Chemistry and Biology, for more details about this subject, we cite Bonheure and Sanchez [3].

In [3], the existence of heteroclinic solution for (1.1)-(1.2) has been studied for some classes of function $a$. More precisely, in that paper the following classes were considered:

Class 1: $a$ is a positive constant.

Class 2: $a$ is a periodic continuous function with

$$
\inf _{t \in \mathbb{R}} a(t)=a_{0}>0 .
$$

Class 3: $a$ is a bounded continuous function and there are $a_{1}, a_{2}>0$ verifying

$$
a_{1} \leq a(t) \leq a_{2} \quad \forall t \in \mathbb{R}
$$

and

$$
a(t) \rightarrow a_{2}, \quad \text { as } \quad|t| \rightarrow+\infty
$$

with $a(t)<a_{2}$ in some set of nonzero measure.

In [10], Gavioli and Sanchez have assumed that $a$ belongs to ensuing class:

Class 4: There is $t_{0}$ such that $a$ is increasing in $\left(-\infty, t_{0}\right], a$ is decreasing in $\left[t_{0},+\infty\right)$, $\lim _{|t| \rightarrow+\infty} a(t)=l>0$, and

$$
\lim _{|t| \rightarrow+\infty}|t|(l-a(t))=0 .
$$

Gavioli [8] has studied the following class

Class 5: There are $0<l<L$ such that

$$
\begin{gathered}
l \leq a(t) \leq L \quad \forall t \in \mathbb{R}, \\
a(t) \rightarrow L \text { as }|t| \rightarrow+\infty,
\end{gathered}
$$

and $L / l$ is suitably bounded from above.

After, Gavioli [9] considered the situation where $a$ is in the class

Class 6: $a \in L^{\infty}(\mathbb{R},[0,+\infty))$ and there are $l>0, S<T$, such that

$$
a(t)=l \text { for } t \notin[S, T] .
$$

Finally, in [12], Spradlin established the existence of heteroclinic for the case where $a$ within class

Class 7: There are $\underline{l}, l>$ such that

$$
a(t) \rightarrow l \text { as }|t| \rightarrow+\infty,
$$

and

$$
\underline{l} \leq a(t) \leq L=\nu \sqrt{l} \underline{l} / \int_{-1}^{1} \sqrt{V(t)} d t
$$

where

$$
\nu=\min \left\{\int_{-1}^{\xi_{-}} \sqrt{V(t)} d t, \int_{\xi_{+}}^{1} \sqrt{V(t)} d t\right\}
$$

with

$$
\xi_{-}=\min \left\{t: t>-1, V^{\prime}(t)=0\right\} \quad \text { and } \quad \xi_{+}=\max \left\{t: t<1, V^{\prime}(t)=0\right\} \text {. }
$$


In the above references, the main idea to get a solution for (1.1)-(1.2) is looking for critical point for the functional $J: H_{l o c}^{1}(\mathbb{R}) \rightarrow[0,+\infty]$ given by

$$
J(x)=\int_{-\infty}^{+\infty}\left(\frac{1}{2}|\dot{x}|^{2}+a(\epsilon t) V(x(t))\right) d t .
$$

In some of them, the existence of critical point was established showing that $J$ possesses a critical point on one of the ensuing sets

$$
\Sigma=\left\{x \in H_{l o c}^{1}(\mathbb{R}): x(-\infty)=-1 \text { and } x(+\infty)=1\right\}
$$

or

$$
W=\left\{x \in H_{l o c}^{1}(\mathbb{R}): x+1 \in H^{1}((-\infty, 0]), x-1 \in H^{1}([0,+\infty))\right\} .
$$

The main tool used is the variational method, more precisely, deformation lemma and minimization techniques.

Motivated by cited references, we intend to study the existence of heteroclinic solution for (1.1)-(1.2) for three new classes of function $a$. Here, we will consider the following classes:

Class 8: $a \in L^{\infty}(\mathbb{R})$ and

$$
\liminf _{|t| \rightarrow \infty} a(t)=a_{\infty}>\inf _{t \in \mathbb{R}} a(t)=a(0)>0 .
$$

This class of functions was introduced by Rabinowitz [14] to study existence of solution for a P.D.E. of the type

$$
-\Delta u+V(\epsilon x) u=f(u), \quad \mathbb{R}^{N} .
$$

This way, throughout this article, we will called it of Rabinowitz's condition.

Class 9: $a$ is asymptotically periodic, that is, there is a continuous periodic function $a_{P}: \mathbb{R} \rightarrow \mathbb{R}$ satisfying:

$$
\left|a(t)-a_{P}(t)\right| \rightarrow 0 \quad \text { as } \quad|t| \rightarrow+\infty
$$

and

$$
0<\inf _{t \in \mathbb{R}} a(t) \leq a(t)<a_{P}(t) \forall t \in \mathbb{R} .
$$

Class 10: $a$ is coercive, that is,

$$
0<\inf _{t \in \mathbb{R}} a(t) \text { and } a(t) \rightarrow+\infty \quad \text { as } \quad|t| \rightarrow+\infty .
$$

Our main result is the following

Theorem 1.1. Assume $\left(V_{1}\right)-\left(V_{4}\right)$ and that a belongs to Class 9 or 10 . Then, for each $\epsilon>0$, problem (1.1)-(1.2) has a solution $x \in H_{\text {loc }}^{1}(\mathbb{R}) \cap C^{2}(\mathbb{R})$ and $x(t) \in(0,1)$ for all $t \in \mathbb{R}$. If a belongs to Class 8 , the existence of solution is established for $\epsilon$ small enough.

In the proof of Theorem 1.1, we explored some arguments used in [3,12]. The basic idea is working with a minimization problem, which will lead us to get a heteroclinic solution for the problem (1.1)-(1.2), for more details, see Sects. 3, 4 and 5 .

Before to conclude this introduction, we would like to cite the papers of Bonheure et al. [5], Bonheure et al. [4], Coti Zelati and Rabinowitz [6], Korman et al. [11], Rabinowitz [15], and their references, where the reader can 
find interesting results about the existence of heteroclinic solutions for related problems.

The plan of the paper is as follows: in Sect. 2, we prove some technical results, which will be useful in the proof of Theorem 1.1. In Sects. 3 and 4 , we study the case where $a$ verifies the Rabinowitz's condition and it is asymptotically periodic respectively, while the coercive case is considered in Sect. 5. In Sect. 6, we make some final considerations.

\section{Technical results}

In this section, we will show some results, which are crucial in the proof of Theorem 1.1. However, we would like to point out that in their proofs it is enough to assume that function $a$ verifies the following condition: There are $l_{0}, l_{1}>0$ such that

$$
l_{0} \leq a(t) \leq l_{1} \quad \forall t \in \mathbb{R} .
$$

To begin with, we observe that from $\left(V_{1}\right)-\left(V_{4}\right)$, there are $C_{1}, C_{2}, \delta>0$ with $C_{1}<C_{2}$, such that

$$
C_{1}(t-1)^{2} \leq V(t) \leq C_{2}(t-1)^{2} \quad \forall t \in(1-\delta, 1+\delta)
$$

and

$$
C_{1}(t+1)^{2} \leq V(t) \leq C_{2}(t+1)^{2} \quad \forall t \in(-1-\delta,-1+\delta) .
$$

In what follows, we will make a modification on function $V$, by assuming that it satisfies the following properties:

$\left(V_{5}\right) \quad V(t)>0$ for all $t \in(-\infty,-1-\delta) \cup(1+\delta,+\infty)$,

$\left(V_{5}\right) \quad V^{\prime}(t) t>0$ for all $t \in(-\infty,-1) \cup(1,+\infty)$,

and

$\left(V_{7}\right) \quad V(t) \rightarrow+\infty \quad$ as $\quad|t| \rightarrow+\infty$.

Hereafter, we will denote by $\tilde{V}$ the new function. This way,

$$
\begin{aligned}
\tilde{V}(t)= & V(t) \forall t \in(-1-\delta, 1+\delta), \tilde{V}^{\prime}(t) t>0 \quad \text { for } \quad|t|>1 \quad \text { and } \tilde{V}(t) \rightarrow+\infty \\
& \text { as }|t| \rightarrow+\infty .
\end{aligned}
$$

Moreover, we denote by $W \subset H_{\text {loc }}^{1}(\mathbb{R})$ the set

$$
W=\left\{x \in H_{l o c}^{1}(\mathbb{R}): x+1 \in H^{1}((-\infty, 0]), x-1 \in H^{1}([0,+\infty))\right\},
$$

and by $J_{\epsilon}: H_{l o c}^{1}(\mathbb{R}) \rightarrow[0,+\infty]$ the functional given by

$$
J_{\epsilon}(x)=\int_{-\infty}^{+\infty}\left(\frac{1}{2}|\dot{x}|^{2}+a(\epsilon t) \tilde{V}(x(t))\right) d t .
$$

From $\left(V_{1}\right)-\left(V_{4}\right)$, more precisely $(2.1)-(2.2)$, it follows that $J_{\epsilon}(x)<+\infty$ for all $x \in W$ and $J_{\epsilon}$ is Fréchet differentiable, in the sense that,

$$
J_{\epsilon}^{\prime}(x) v=\int_{-\infty}^{+\infty}(\dot{x} \dot{v}+a(\epsilon t) \tilde{V}(x(t)) v(t)) d t \quad \forall x \in W \quad \text { and } \quad v \in H^{1}(\mathbb{R}) .
$$


In the sequel, we say that $\left(x_{n}\right)$ is a $(P S)_{c}$ sequence for $J_{\epsilon}$, if $\left(x_{n}\right) \subset W$ with

$$
J_{\epsilon}\left(x_{n}\right) \rightarrow c \text { and }\left\|J_{\epsilon}^{\prime}\left(x_{n}\right)\right\|_{*} \rightarrow 0 \quad \text { as } \quad n \rightarrow \infty
$$

where

$$
\left\|J_{\epsilon}^{\prime}(x)\right\|_{*}=\sup \left\{J_{\epsilon}^{\prime}(x) v: v \in H^{1}(\mathbb{R}),\|v\|=1\right\},
$$

and \|\| denotes the usual norm in $H^{1}(\mathbb{R})$.

The next two lemmas can be found in [12], however for reader's convenience we will write their proofs.

Lemma 2.1. If $x \in H_{l o c}^{1}(\mathbb{R})$ and $J_{\epsilon}(x)<\infty$, then

$$
x(t) \rightarrow-1 \quad \text { or } \quad x(t) \rightarrow 1 \quad \text { as } \quad t \rightarrow-\infty
$$

and

$$
x(t) \rightarrow 1 \quad \text { or } \quad x(t) \rightarrow-1 \quad \text { as } \quad t \rightarrow+\infty .
$$

More precisely,

$$
x+1 \in H^{1}((-\infty, 0]) \quad \text { or } \quad x-1 \in H^{1}((-\infty, 0])
$$

and

$$
x+1 \in H^{1}([0,+\infty)) \quad \text { or } \quad x-1 \in H^{1}([0,+\infty)) .
$$

Proof. Suppose the lemma is false. Then, there is $x \in H_{l o c}^{1}(\mathbb{R})$ with $J_{\epsilon}(x)<\infty$, $\eta>0$ and a sequence $\left(t_{n}\right)$ with $\left|t_{n}\right| \rightarrow+\infty$ as $n \rightarrow+\infty$ such that

$$
x\left(t_{n}\right) \in(-\infty,-1-\eta) \cup(-1+\eta, 1-\eta) \cup(1+\eta, \infty) .
$$

Let

$$
d=\{\tilde{V}(t): t \in(-\infty,-1-\eta / 2) \cup(-1+\eta / 2,1-\eta / 2) \cup(1+\eta / 2,+\infty)\}>0 .
$$

We can assume, without loss of generality, $t_{n} \rightarrow+\infty$ and $t_{n+1} \geq t_{n}+1$ for all $n \in \mathbb{N}$. If

$x(t) \in(-\infty,-1-\eta / 2) \cup(-1+\eta / 2,1-\eta / 2) \cup(1+\eta / 2,+\infty) \forall t \in\left[t_{n}, t_{n+1}\right]$, we have that

$$
\int_{t_{n}}^{t_{n}+1} a(\epsilon t) \tilde{V}(x(t)) d t \geq l_{0} d \forall n \in \mathbb{N} .
$$

Otherwise, there exists $t^{*} \in\left[t_{n}, t_{n}+1\right]$ with $\left|x\left(t^{*}\right)-x\left(t_{n}\right)\right| \geq \eta / 2$. Thereby,

$$
\eta / 2 \leq\left|x\left(t^{*}\right)-x\left(t_{n}\right)\right| \leq \int_{t_{n}}^{t^{*}}|\dot{x}| d t \leq \sqrt{t^{*}-t_{n}}\left(\int_{t_{n}}^{t^{*}}|\dot{x}|^{2} d t\right)^{\frac{1}{2}}
$$

from where it follows that

$$
\int_{t_{n}}^{t_{n}+1}|\dot{x}|^{2} d t \geq \eta^{2} / 4
$$

From (2.5) and (2.6),

$$
\int_{t_{n}}^{t_{n}+1}\left(\frac{1}{2}|\dot{x}|^{2}+a(\epsilon t) \tilde{V}(x(t))\right) d t \geq \min \left\{l_{0} d, \eta^{2} / 4\right\}
$$


and so,

$$
J_{\epsilon}(x) \geq \sum_{n=1}^{+\infty} \int_{t_{n}}^{t_{n}+1}\left(\frac{1}{2}|\dot{x}|^{2}+a(\epsilon t) \tilde{V}(x(t))\right) d t=+\infty
$$

which is a contradiction, because by hypothesis $J_{\epsilon}(x)<\infty$. Then,

$$
x(t) \rightarrow-1 \quad \text { or } \quad x(t) \rightarrow 1 \quad \text { as } \quad t \rightarrow+\infty .
$$

The same argument works to prove that

$$
x(t) \rightarrow-1 \quad \text { or } \quad x(t) \rightarrow 1 \quad \text { as } \quad t \rightarrow-\infty .
$$

By (2.1)-(2.2), if $x(t) \rightarrow 1$ as $t \rightarrow+\infty$, there is $T>0$ such that

$$
\begin{aligned}
\int_{T}^{+\infty}(x(t)-1)^{2} & \leq \int_{T}^{+\infty} \frac{\tilde{V}(x(t))}{C_{1}} d t \leq \frac{1}{l_{0} C_{1}} \int_{T}^{+\infty} a(\epsilon t) \tilde{V}(x(t)) d t \\
& \leq \frac{1}{l_{0} C_{1}} J_{\epsilon}(x)<\infty .
\end{aligned}
$$

The last inequality yields $x-1 \in H^{1}([0,+\infty))$. Analogous approach can be repeated to the cases

$$
\begin{aligned}
& x(t) \rightarrow-1 \text { as } t \rightarrow+\infty, x(t) \rightarrow 1 \text { as } t \rightarrow-\infty \\
& \text { and } x(t) \rightarrow-1 \text { as } t \rightarrow-\infty .
\end{aligned}
$$

The next lemma will be used to study the convergence of the PalaisSmale sequences associated with $J_{\epsilon}$.

Lemma 2.2. Let $A, T>0$. There is $B>0$, such that if $x \in H_{\text {loc }}^{1}(\mathbb{R})$ with $J_{\epsilon}(x) \leq A$, then $\|x\|_{H^{1}([-T, T])} \leq B$.

Proof. First of all, note that

$$
\int_{-T}^{T}|\dot{x}|^{2} d t \leq 2 A
$$

By coercivity of $\tilde{V}$, there exists $C>0$ such that

$$
\tilde{V}(t)>\frac{A}{l_{0} T} \quad \text { for } \quad|t| \geq C .
$$

Since

$$
\int_{-T}^{T} a(\epsilon t) \tilde{V}(x(t)) d t \leq A,
$$

there is $t^{*} \in[-T, T]$ such that $\tilde{V}\left(x\left(t^{*}\right)\right) \leq \frac{A}{2 T}$ and $\left|x\left(t^{*}\right)\right| \leq C$. Hence, for all $s \in[-T, T]$,

$$
\begin{aligned}
|x(s)| & \leq\left|x\left(t^{*}\right)\right|+\left|\int_{\min \left\{s, t^{*}\right\}}^{\max \left\{s, t^{*}\right\}} \dot{x}(t) d t\right| \leq\left|x\left(t^{*}\right)\right|+\left.\left.\sqrt{\left|s-t^{*}\right|}\left|\int_{\min \left\{s, t^{*}\right\}}^{\max \left\{s, t^{*}\right\}}\right| \dot{x}\right|^{2} d t\right|^{\frac{1}{2}} \\
& \leq C+2 \sqrt{T A}
\end{aligned}
$$


showing that

$$
\|x\|_{\infty} \leq C+2 \sqrt{A T} .
$$

\section{Existence of solution for Rabinowitz's condition}

In this section, we intend to prove Theorem 1.1, by assuming that $a$ verifies the Rabinowitz's condition.

In what follows, we denote by $\mathcal{B}_{\epsilon}, \mathcal{B}_{0}$ and $\mathcal{B}_{\infty}$ the following real numbers

$$
\begin{aligned}
& \mathcal{B}_{\epsilon}=\inf \left\{J_{\epsilon}(x): x \in W\right\}, \\
& \mathcal{B}_{0}=\inf \left\{J_{0}(x): x \in W\right\},
\end{aligned}
$$

and

$$
\mathcal{B}_{\infty}=\inf \left\{J_{\infty}(x): x \in W\right\},
$$

where $J_{\infty}: H_{\text {loc }}^{1}(\mathbb{R}) \rightarrow[0,+\infty]$ is the functional given by

$$
J_{\infty}(x)=\int_{-\infty}^{+\infty}\left(\frac{1}{2}|\dot{x}|^{2}+a_{\infty} \tilde{V}(x(t))\right) d t .
$$

Related to the above numbers, we have the ensuing result

Lemma 3.1. The numbers $\mathcal{B}_{\epsilon}, \mathcal{B}_{0}$ and $\mathcal{B}_{\infty}$ verify

$$
\mathcal{B}_{0}<\mathcal{B}_{\infty} \quad \text { and } \quad \lim _{\epsilon \rightarrow 0} \mathcal{B}_{\epsilon}=\mathcal{B}_{0} .
$$

Proof. In what follows, we denote by $w_{0}, w_{\infty} \in W$ the functions that verify

$$
\left\{\begin{array}{l}
\ddot{w}_{0}(t)=a(0) V^{\prime}\left(w_{0}(t)\right), \quad t \in \mathbb{R}, \\
w_{0}(t) \in(-1,1) \forall t \in \mathbb{R}, \\
w_{0}(t) \rightarrow-1 \text { as } t \rightarrow-\infty, w_{0}(t) \rightarrow 1 \text { as } t \rightarrow+\infty,
\end{array}\right.
$$

and

$$
\left\{\begin{array}{l}
\ddot{w}_{\infty}(t)=a_{\infty} V^{\prime}\left(w_{\infty}(t)\right), \quad t \in \mathbb{R} \\
w_{\infty}(t) \in(-1,1) \forall t \in \mathbb{R}, \\
w_{\infty}(t) \rightarrow-1 \text { as } t \rightarrow-\infty, w_{\infty}(t) \rightarrow 1 \text { as } t \rightarrow+\infty
\end{array}\right.
$$

with

$$
\mathcal{B}_{0}=J_{0}\left(w_{0}\right) \quad \text { and } \quad \mathcal{B}_{\infty}=J_{\infty}\left(w_{\infty}\right) .
$$

The existence of $w_{0}$ and $w_{\infty}$ was established in [3].

By hypothesis $a_{0}<a_{\infty}$, then

$$
\mathcal{B}_{0} \leq J_{0}\left(w_{\infty}\right)<J_{\infty}\left(w_{\infty}\right)=\mathcal{B}_{\infty},
$$

showing the first part of the lemma. For the second part, we begin observing that

$$
J_{0}(w) \leq J_{\epsilon}(w) \forall w \in W
$$

Consequently,

$$
\mathcal{B}_{0} \leq \mathcal{B}_{\epsilon} \forall \epsilon>0
$$


leading to

$$
\mathcal{B}_{0} \leq \liminf _{\epsilon \rightarrow 0} \mathcal{B}_{\epsilon}
$$

On the other hand, since $w_{0} \in W$,

$$
\mathcal{B}_{\epsilon} \leq J_{\epsilon}\left(w_{0}\right)=\int_{-\infty}^{+\infty}\left(\frac{1}{2}\left|\dot{w}_{0}\right|^{2}+a(\epsilon t) \tilde{V}\left(w_{0}(t)\right)\right) d t .
$$

Using Lebesgue's Theorem, we deduce that

$$
\limsup _{\epsilon \rightarrow 0} \mathcal{B}_{\epsilon} \leq \int_{-\infty}^{+\infty}\left(\frac{1}{2}\left|\dot{w}_{0}\right|^{2}+a(0) \tilde{V}\left(w_{0}(t)\right)\right) d t=J_{0}\left(w_{0}\right)=\mathcal{B}_{0} .
$$

From (3.1)-(3.2),

$$
\lim _{\epsilon \rightarrow 0} \mathcal{B}_{\epsilon}=\mathcal{B}_{0}
$$

The next lemma establishes that minimum points of $J$ on $W$ are in fact solutions for (1.1)-(1.2).

Lemma 3.2. If $x \in W$ verifies $J_{\epsilon}(x)=\mathcal{B}_{\epsilon}$, then $x$ solves problem (1.1)-(1.2) and $x(t) \in(-1,1)$ for all $t \in \mathbb{R}$.

Proof. Since $J_{\epsilon}(x)=\mathcal{B}_{\epsilon}$, it is possible to prove that

$$
J_{\epsilon}^{\prime}(x) v=0 \quad \forall v \in H^{1}(\mathbb{R}),
$$

showing that $x$ is a critical point of $J_{\epsilon}$. Therefore, $x$ is a solution of O.D.E.

$$
\ddot{x}(t)=a(\epsilon t) \tilde{V}^{\prime}(x(t)), \quad t \in \mathbb{R} .
$$

Moreover, by $x \in W$, one have

$$
x(t) \rightarrow-1 \quad \text { as } \quad t \rightarrow-\infty, x(t) \rightarrow 1 \quad \text { as } \quad t \rightarrow+\infty .
$$

Now, we will prove that

$$
x(t) \in(-1,1) \forall t \in \mathbb{R} .
$$

If $x(t)>1$ for some $t \in \mathbb{R}$, then let $t_{0} \in \mathbb{R}$ with $x\left(t_{0}\right)=\max _{t \in \mathbb{R}} x(t)>1$. Thereby,

$$
\ddot{x}\left(t_{0}\right) \leq 0 \quad \text { and } \quad \tilde{V}^{\prime}\left(x\left(t_{0}\right)\right)>0,
$$

which is an absurd. Thus $x(t)<1$ for all $t \in \mathbb{R}$. The same type of argument works to show that $x(t)>-1$ for all $t \in \mathbb{R}$. From the above information, we can conclude that $x$ is a solution for original problem (1.1)-(1.2), because

$$
\tilde{V}(x(t))=V(x(t)) \forall t \in \mathbb{R},
$$

finishing the proof of lemma.

The next result shows that associated with $\mathcal{B}_{\epsilon}$, we have a Palais-Smale sequence for $J_{\epsilon}$.

Lemma 3.3. There is a $(P S)_{\mathcal{B}_{\epsilon}}$ sequence for $J_{\epsilon}$. 
Proof. Since $J_{\epsilon}$ is bounded from below, there is $\left(x_{n}\right) \subset W$ such that

$$
J_{\epsilon}\left(x_{n}\right) \rightarrow \mathcal{B}_{\epsilon} \text { as } n \rightarrow+\infty .
$$

Now, it is easy to check that if $x, z \in W$, then $x-z \in H^{1}(\mathbb{R})$. Therefore, we can define on $W$ the metric $\rho: W \times W \rightarrow[0+\infty)$ given by

$$
\rho(x, z)=\|x-z\|,
$$

where \|\| denotes the usual norm in $H^{1}(\mathbb{R})$. A direct computation gives that $(W, \rho)$ is a complete metric space. Since $J_{\epsilon}$ is lower semicontinuous and bounded from below on $(W, \rho)$, by Ekeland's Variational Principle there is $\left(u_{n}\right) \subset W$ verifying

$$
\left\|x_{n}-u_{n}\right\|=o_{n}(1)
$$

with

$$
J_{\epsilon}\left(u_{n}\right) \rightarrow \mathcal{B}_{\epsilon} \text { and } J_{\epsilon}^{\prime}\left(u_{n}\right) \rightarrow 0 \text { as } n \rightarrow+\infty,
$$

implying that $\left(u_{n}\right)$ is a $(P S)_{\mathcal{B}_{\epsilon}}$ sequence for $J_{\epsilon}$.

The next lemma is crucial in our approach and its proof can be found in $[12]$.

Lemma 3.4. Let $x_{0}, x_{1} \in(-1,1), x_{0}<x_{1}, t_{0}<t_{1}$ and $x \in H^{1}\left(\left[t_{0}, t_{1}\right]\right)$ with $x\left(t_{0}\right)=x_{0}$ and $x\left(t_{1}\right)=x_{1}$. Then,

$$
\int_{t_{0}}^{t_{1}}\left(\frac{1}{2}|\dot{x}|^{2}+a_{\infty} V(x(t))\right) d t \geq \int_{w_{\infty}^{-1}\left(x_{0}\right)}^{w_{\infty}^{-1}\left(x_{1}\right)}\left(\frac{1}{2}\left|w_{\infty}\right|^{2}+a_{\infty} V\left(w_{\infty}(t)\right)\right) d t,
$$

where $w_{\infty}$ was given in the proof of Lemma 3.1 .

The main result this section can be stated as follows

Theorem 3.1. Assume that $\left(V_{1}\right)-\left(V_{4}\right)$ hold. If a belongs to Class 8 , there is $\epsilon^{*}>0$, such that problem $(1.1)-(1.2)$ has a solution $x \in H_{\text {loc }}^{1}(\mathbb{R}) \cap C^{2}(\mathbb{R})$ for all $\epsilon \in\left(0, \epsilon^{*}\right)$. Moreover, $x(t) \in(0,1)$ for all $t \in \mathbb{R}$.

Proof. First of all, by Lemma 3.2, we see that to prove Theorem 1.1, it is enough to show that there exists $\epsilon^{*}>0$ such that $\mathcal{B}_{\epsilon}$ is achieved for all $\epsilon \in$ $\left[0, \epsilon^{*}\right)$.

To this end, we will set for each $\tau>0$ the real number

$$
\Lambda_{\tau}=\int_{w_{\infty}^{-1}(-1+\tau)}^{w_{\infty}^{-1}(1-\tau)}\left(\frac{1}{2}\left|w_{\infty}\right|^{2}+a_{\infty} V\left(w_{\infty}(t)\right)\right) d t
$$

where $w_{\infty} \in W, w_{\infty}(t) \in(-1,1)$ for all $t \in \mathbb{R}$ and $J_{\infty}\left(w_{\infty}\right)=\mathcal{B}_{\infty}$. By a routine calculus,

$$
\Lambda_{\tau} \rightarrow \mathcal{B}_{\infty} \text { as } \tau \rightarrow 0
$$

In the last limit, we have used that $\lim _{t \rightarrow+\infty} w_{\infty}(t)=1$ and $\lim _{t \rightarrow-\infty} w_{\infty}(t)=-1$.

The inequality $\mathcal{B}_{0}<\mathcal{B}_{\infty}$ in conjunction with (3.3) implies that there is $\tau>0$ small enough verifying

$$
\left(\frac{a_{\infty}-\tau}{a_{\infty}}\right) \Lambda_{\tau}>\mathcal{B}_{0}
$$


From Lemma 3.1, we know that $\lim _{\epsilon \rightarrow 0} \mathcal{B}_{\epsilon}=\mathcal{B}_{0}$. Then, this limit together with the last inequality yields there is $\epsilon^{*}>0$ such that

$$
\mathcal{B}_{\epsilon}<\left(\frac{a_{\infty}-\tau}{a_{\infty}}\right) \Lambda_{\tau}, \quad \forall \epsilon \in\left[0, \epsilon^{*}\right) .
$$

To prove that $\mathcal{B}_{\epsilon}$ is achieved for each $\epsilon \in\left[0, \epsilon^{*}\right)$, we begin recalling that from Lemma 3.3, there is a $(P S)_{\mathcal{B}_{\epsilon}}$ sequence for $J_{\epsilon}$, that is, there exists $\left(x_{n}^{\epsilon}\right) \subset$ $W$ such that

$$
J_{\epsilon}\left(x_{n}^{\epsilon}\right) \rightarrow \mathcal{B}_{\epsilon} \text { and }\left\|J_{\epsilon}^{\prime}\left(x_{n}^{\epsilon}\right)\right\|_{*} \rightarrow 0 \quad \text { as } n \rightarrow+\infty .
$$

From this,

$$
J_{\epsilon}\left(x_{n}^{\epsilon}\right) \leq A^{\epsilon}=\sup _{n} J_{\epsilon}\left(x_{n}^{\epsilon}\right) \forall n \in \mathbb{N} .
$$

By Lemma 2.2, for each $T>0$, there is $B=B(T, A)>0$ such that

$$
\left\|x_{n}^{\epsilon}\right\|_{H^{1}([-T, T])} \leq B \quad \forall n \in \mathbb{N} .
$$

Hence, there is a subsequence of $\left(x_{n}^{\epsilon}\right)$, still denoted by itself, and $x^{\epsilon} \in H_{l o c}^{1}(\mathbb{R})$ verifying

$$
x_{n}^{\epsilon} \rightarrow x^{\epsilon} \text { uniformly in }[-T, T] \text { and } x_{n}^{\epsilon} \rightarrow x^{\epsilon} \text { in } H^{1}([-T, T]) \forall T>0 .
$$

Combining these limits with the fact that $J_{\epsilon}$ is lower semicontinuous, we also derive that

$$
J_{\epsilon}\left(x^{\epsilon}\right) \leq \mathcal{B}_{\epsilon} .
$$

Next, we will show that $J_{\epsilon}^{\prime}\left(x^{\epsilon}\right)=0$. To see why, note that for each $v \in C_{0}^{\infty}(\mathbb{R})$ fixed, we have that $J_{\epsilon}^{\prime}\left(x_{n}^{\epsilon}\right) v=o_{n}(1)$. Then,

$$
\int_{\alpha}^{\beta} \dot{x}_{n}^{\epsilon} \dot{v} d t+\int_{\alpha}^{\beta} a(\epsilon t) \tilde{V}^{\prime}\left(x_{n}^{\epsilon}(t)\right) v(t) d t=o_{n}(1),
$$

where supp $v \subset[\alpha, \beta]$. Letting $n \rightarrow+\infty$, we get

$$
\int_{\alpha}^{\beta} \dot{x^{\epsilon}} \dot{v} d t+\int_{\alpha}^{\beta} a(\epsilon t) \tilde{V}^{\prime}\left(x^{\epsilon}(t)\right) v(t) d t=0,
$$

implying that $x^{\epsilon}$ is a solution of equation O.D.E.

$$
\ddot{x} \epsilon(t)=a(\epsilon t) \tilde{V}^{\prime}\left(x^{\epsilon}(t)\right),
$$

and so,

$$
J_{\epsilon}^{\prime}\left(x^{\epsilon}\right)=0 .
$$

Moreover, by Fatous' Lemma $J_{\epsilon}\left(x^{\epsilon}\right)<+\infty$. Consequently, by Lemma 2.1

$$
x^{\epsilon}(t) \rightarrow-1 \text { or } x^{\epsilon}(t) \rightarrow 1 \quad \text { as } t \rightarrow-\infty
$$

and

$$
x^{\epsilon}(t) \rightarrow 1 \text { or } x^{\epsilon}(t) \rightarrow-1 \quad \text { as } t \rightarrow+\infty .
$$

Our next step is showing that the limit below

$$
x^{\epsilon}(t) \rightarrow-1 \quad \text { as } t \rightarrow+\infty
$$

does not hold. 
Now, by $\left(a_{7}\right)$, let $T^{\epsilon}>0$ be large enough so that $a(\epsilon t) \geq a_{\infty}-\tau$ on $\left[T^{\epsilon},+\infty\right)$ and $x\left(T^{\epsilon}\right)<-1+\tau$. Let $n$ be a large enough so that $x_{n}^{\epsilon}(T)<-1+\tau$. Let $T^{\epsilon}<\alpha^{\epsilon}<\beta^{\epsilon}$ with $x_{n}^{\epsilon}\left(\alpha^{\epsilon}\right)=-1+\tau$ and $x_{n}^{\epsilon}\left(\beta^{\epsilon}\right)=1-\tau$. By Lemma 3.4, we get the inequality

$$
J_{\epsilon}\left(x_{n}^{\epsilon}\right) \geq\left(\frac{a_{\infty}-\tau}{a_{\infty}}\right) \int_{\alpha^{\epsilon}}^{\beta^{\epsilon}}\left(\frac{1}{2}\left|\dot{x}_{n}^{\epsilon}\right|^{2}+a_{\infty} \tilde{V}\left(x_{n}^{\epsilon}(t)\right)\right) d t \geq\left(\frac{a_{\infty}-\tau}{a_{\infty}}\right) \Lambda_{\tau},
$$

which gives

$$
\mathcal{B}_{\epsilon}=\lim _{n \rightarrow+\infty} J_{\epsilon}\left(x_{n}^{\epsilon}\right) \geq\left(\frac{a_{\infty}-\tau}{a_{\infty}}\right) \Lambda_{\tau},
$$

contradicting (3.4). This way,

$$
x^{\epsilon}(t) \rightarrow 1 \quad \text { as } t \rightarrow+\infty \text {. }
$$

A similar argument can be used to show that

$$
x^{\epsilon}(t) \rightarrow-1 \quad \text { as } t \rightarrow-\infty .
$$

As in the proof of Lemma 2.1, we derive that $x^{\epsilon}+1 \in H^{1}((-\infty, 0])$ and $x^{\epsilon}-1 \in H^{1}\left([0,+\infty)\right.$. Then, $x^{\epsilon} \in W$, and by $(3.5), J_{\epsilon}\left(x^{\epsilon}\right)=\mathcal{B}_{\epsilon}$ finishing the proof.

\section{Existence of solution for the asymptotically periodic case}

In this section, we intend to prove the existence of solution for (1.1)-(1.2), by assuming that $a$ is asymptotically periodic.

The main result in this section is the following

Theorem 4.1. Assume that $\left(V_{1}\right)-\left(V_{4}\right)$ hold. If a belongs to Class 9 , problem (1.1)-(1.2) has a solution $x \in H_{\text {loc }}^{1}(\mathbb{R}) \cap C^{2}(\mathbb{R})$ for each $\epsilon>0$. Moreover, $x(t) \in(0,1)$ for all $t \in \mathbb{R}$.

In the proof of Theorem 4.1, without loss of generality, we assume that $\epsilon=1$. Moreover, we will use the fact that problem (1.1)-(1.2) has an increasing solution $w_{P} \in H_{\text {loc }}^{1}(\mathbb{R}) \cap C^{2}(\mathbb{R})$ with $w_{P} \in W$ and $J_{P}\left(w_{P}\right)=\mathcal{B}_{P}$, where $J_{P}: H_{l o c}^{1}(\mathbb{R}) \rightarrow[0,+\infty]$ is the functional given by

$$
J_{P}(x)=\int_{-\infty}^{+\infty}\left(\frac{1}{2}|\dot{x}|^{2}+a_{P}(t) \tilde{V}(x(t))\right) d t .
$$

and

$$
\mathcal{B}_{P}=\inf \left\{J_{P}(x): x \in W\right\} .
$$

The existence of $w_{P}$ can be seen in [3].

In the sequel, we denote by $J: H_{l o c}^{1}(\mathbb{R}) \rightarrow[0,+\infty]$ the functional given by

$$
J(x)=\int_{-\infty}^{+\infty}\left(\frac{1}{2}|\dot{x}|^{2}+a(t) \tilde{V}(x(t))\right) d t
$$

and by $\mathcal{B}$, the real number given by

$$
\mathcal{B}=\inf \{J(x): x \in W\} .
$$


Here, we would like point out that all results proved in Sect. 2 are true for functionals $J$ and $J_{P}$. Moreover, from $\left(a_{9}\right)$, we also have

$$
\mathcal{B}<\mathcal{B}_{P}
$$

\section{Proof of Theorem 4.1}

As in the proof of Theorem 3.1, our main goal is to show that $\mathcal{B}$ is achieved on $W$. Hereafter, $a_{0}=\inf _{t \in \mathbb{R}} a(t)>0$ and we fix $\delta>0$ such that

$$
\mathcal{B}+\delta<\mathcal{B}_{P}
$$

Moreover, we also fix $M=M(\delta)>0$ such that

$$
\left|a(t)-a_{P}(t)\right|<\frac{\delta a_{0}}{4 \mathcal{B}} \quad \text { for }|t|>M
$$

and $\gamma \in(0,1)$ verifying

$$
\tilde{V}(z)<\frac{\delta}{16 M\left\|a_{P}\right\|_{\infty}} \forall z \in[-1,-1+\gamma] \cup[1-\gamma, 1] .
$$

Claim 4.1. For each $\gamma \in(0,1)$, there are sequences $\left(U_{n}\right) \subset W,\left(s_{n}\right),\left(t_{n}\right) \subset \mathbb{R}$ with $s_{n}<t_{n}$ satisfying:

$$
\begin{gathered}
J\left(U_{n}\right) \rightarrow \mathcal{B} \quad \text { as } \quad n \rightarrow+\infty, \\
U_{n}(t) \in[-1,-1+\gamma] \forall t \in\left(-\infty, s_{n}\right], \\
U_{n}(t) \in[1-\gamma, 1] \forall t \in\left[t_{n},+\infty\right), \\
U_{n}(t) \in[-1+\gamma, 1-\gamma] \forall t \in\left[s_{n}, t_{n}\right], \\
U_{n}\left(t_{n}\right)=1-\gamma, \quad U_{n}\left(s_{n}\right)=-1+\gamma,
\end{gathered}
$$

and

$$
\left(t_{n}-s_{n}\right) \text { is bounded in } \mathbb{R} \text {. }
$$

The existence of the above sequences can be found in [3], however for reader's convenience, we will write its proof. Let $\left(u_{n}\right) \subset W$ be a minimizing sequence for $J$, that is,

$$
J\left(u_{n}\right) \rightarrow \mathcal{B}
$$

By setting the sequence

$$
v_{n}(t)=\max \left\{-1, \min \left\{u_{n}(t), 1\right\}\right\},
$$

a direct computation implies that $\left(v_{n}\right) \subset W$ and

$$
J\left(v_{n}\right) \leq J\left(u_{n}\right) \forall n \in \mathbb{N} .
$$

Therefore, we can assume without loss of generality

$$
-1 \leq u_{n}(t) \leq 1 \quad \forall t \in \mathbb{R} .
$$

Assuming that $\gamma \in(0,1)$ is small enough, it is possible to find an interval $\left[s_{n}, t_{n}\right]$ such that $u_{n}\left(s_{n}\right)=-1+\gamma, u_{n}\left(t_{n}\right)=1-\gamma$ and

$$
-1+\gamma \leq u_{n}(t) \leq 1-\gamma \forall t \in\left[s_{n}, t_{n}\right] \text {. }
$$


Using the above notations, we set a new sequence $\left(U_{n}\right)$ given by

$$
U_{n}(t)= \begin{cases}\min \left\{-1+\gamma, u_{n}(t)\right\}, & \text { if } t \leq s_{n}, \\ u_{n}(t), & \text { if } s_{n} \leq t \leq t_{n}, \\ \max \left\{1-\gamma, u_{n}(t)\right\}, & \text { if } t \geq t_{n} .\end{cases}
$$

A straightforward computation gives

$$
\int_{\mathbb{R}}\left|\dot{U}_{n}\right|^{2} d t \leq \int_{\mathbb{R}}\left|\dot{u}_{n}\right|^{2} d t \quad \forall n \in \mathbb{N} .
$$

Moreover, by $\left(V_{6}\right)$, we also have

$$
\tilde{V}\left(U_{n}(t)\right) \leq \tilde{V}\left(u_{n}(t)\right) \forall t \in \mathbb{R} \quad \text { and } \quad n \in \mathbb{N},
$$

leading to

$$
\int_{\mathbb{R}} a(t) \tilde{V}\left(U_{n}(t)\right) d t \leq \int_{\mathbb{R}} a(t) \tilde{V}\left(u_{n}(t)\right) d t \forall n \in \mathbb{N}
$$

Thus,

$$
J\left(U_{n}\right) \leq J\left(u_{n}\right) \quad \forall n \in \mathbb{N}
$$

Gathering these information, we deduce that $\left(U_{n}\right)$ verifies $(i)-(v)$. Now, the boundedness of $\left(t_{n}-s_{n}\right)$ follows of the inequality below

$$
J\left(U_{n}\right) \geq a_{0}\left(\min _{z \in[-1+\gamma, 1-\gamma]} V(z)\right)\left(t_{n}-s_{n}\right) \quad \forall n \in \mathbb{N},
$$

because $\min _{z \in[-1+\gamma, 1-\gamma]} V(z)>0$ and $a_{0}=\inf _{t \in \mathbb{R}} a(t)>0$.

Now, applying the Ekeland's variational principle, we will find a new sequence, still denoted by $\left(U_{n}\right)$, which verifies:

$$
\begin{gathered}
J\left(U_{n}\right) \rightarrow \mathcal{B} \text { and } J^{\prime}\left(U_{n}\right) \rightarrow 0 \text { as } n \rightarrow+\infty, \\
U_{n}(t) \in[-1-1 / n,-1+\gamma+1 / n] \quad \forall t \in\left(-\infty, s_{n}\right], \\
U_{n}(t) \in[1-\gamma-1 / n, 1+1 / n] \forall t \in\left[t_{n},+\infty\right), \\
U_{n}(t) \in[-1+\gamma-1 / n, 1-\gamma+1 / n] \forall t \in\left[s_{n}, t_{n}\right], \\
U_{n}\left(t_{n}\right)=1-\gamma+o_{n}(1), U_{n}\left(s_{n}\right)=-1+\gamma+o_{n}(1)
\end{gathered}
$$

and

$$
\left(t_{n}-s_{n}\right) \text { is bounded in } \mathbb{R} \text {. }
$$

A direct computation shows that for some subsequence of $\left(U_{n}\right)$, still denoted by itself, there is $U \in C(\mathbb{R}) \cap H_{\text {loc }}^{1}(\mathbb{R})$ such that

$$
U_{n} \rightarrow U \text { uniformly in }[-T, T] \text { and } U_{n} \rightarrow U \text { in } H^{1}([-T, T]) \forall T>0 \text {. }
$$

As in the proof of Theorem 3.1, we see that

$$
J(U) \leq \mathcal{B} \text { and } J^{\prime}(U)=0 .
$$

This way, the theorem follows provided that $U \in W$. To show this fact, we make the following claim 
Claim 4.2. The sequence $\left(s_{n}\right)$ is bounded.

Indeed, if the claim is not true, we must have for some subsequence,

$$
s_{n} \rightarrow+\infty \text { or } s_{n} \rightarrow-\infty \text {. }
$$

Using the above limits, we deduce that

$$
U(t) \in[-1,-1+\gamma] \cup[1-\gamma, 1] \quad \forall t \in \mathbb{R} .
$$

Thus by (4.4),

$$
\tilde{V}(U(t))<\frac{\delta}{16 M\left\|a_{P}\right\|_{\infty}} \forall t \in \mathbb{R}
$$

Note that

$$
\begin{aligned}
J\left(U_{n}\right)= & J_{P}\left(U_{n}\right)+\int_{\mathbb{R}}\left(a(t)-a_{P}(t)\right) \tilde{V}\left(U_{n}(t)\right) d t \geq \mathcal{B}_{P} \\
& +\int_{\mathbb{R}}\left(a(t)-a_{P}(t)\right) \tilde{V}\left(U_{n}(t)\right) d t .
\end{aligned}
$$

Using (4.3), we derive that

$$
\int_{|t|>M}\left|a(t)-a_{P}(t)\right| \tilde{V}\left(U_{n}(t)\right) d t \leq \frac{\delta a_{0}}{4 \mathcal{B}} \int_{|t|>M} \tilde{V}\left(U_{n}(t)\right) d t \leq \frac{\delta}{4 \mathcal{B}} J\left(U_{n}\right),
$$

from where it follows that

$$
\limsup _{n \rightarrow+\infty} \int_{|t|>M}\left|a(t)-a_{P}(t)\right| \tilde{V}\left(U_{n}(t)\right) d t \leq \frac{\delta}{4} .
$$

Now, by (4.5) and (4.6),

$$
\begin{aligned}
& \lim _{n \rightarrow+\infty} \int_{|t| \leq M}\left|a(t)-a_{P}(t)\right| \tilde{V}\left(U_{n}(t)\right) d t \\
& \quad=\lim _{n \rightarrow+\infty} \int_{|t| \leq M}\left|a(t)-a_{P}(t)\right| \tilde{V}(U(t)) d t \leq \frac{\delta}{4} .
\end{aligned}
$$

Consequently, from (4.8) and (4.9),

$$
\limsup _{n \rightarrow+\infty} \int_{\mathbb{R}}\left|a(t)-a_{P}(t)\right| \tilde{V}\left(U_{n}(t)\right) d t \leq \frac{\delta}{2}
$$

Thereby, there is $n_{0} \in \mathbb{N}$ such that

$$
\int_{\mathbb{R}}\left|a(t)-a_{P}(t)\right| \tilde{V}\left(U_{n}(t)\right) d t<\delta \quad \forall n \geq n_{0} .
$$

Combining (4.7) with (4.10), we derive

$$
J\left(U_{n}\right)=J_{P}\left(U_{n}\right)+\int_{\mathbb{R}}\left(a(t)-a_{P}(t)\right) \tilde{V}\left(U_{n}(t)\right) d t \geq \mathcal{B}_{P}-\delta \quad \forall n \geq n_{0},
$$

that is,

$$
J\left(U_{n}\right) \geq \mathcal{B}_{P}-\delta \quad \forall n \geq n_{0}
$$

Taking the limit as $n \rightarrow+\infty$ in the last inequality, we obtain the estimate

$$
\mathcal{B}>\mathcal{B}_{P}-\delta
$$

which contradicts (4.2). 
The boundedness of $\left(s_{n}\right)$ implies that $\left(t_{n}\right)$ is also bounded, thus we can assume without of generality, that there are $t, s \in \mathbb{R}$ verifying

$$
s_{n} \rightarrow s \text { and } t_{n} \rightarrow t \text { as } n \rightarrow+\infty \text {. }
$$

This way,

$$
U(z) \in[-1,-1+\gamma] \forall z \in(-\infty, s) \text { and } U(z) \in[1-\gamma, 1] \forall z \in(t,+\infty) .
$$

The above information together with Lemma 2.1 gives

$$
U(z) \rightarrow 1 \text { as } z \rightarrow+\infty \text { and } U(z) \rightarrow-1 \text { as } z \rightarrow-\infty
$$

This finishes the proof of Theorem 4.1 .

\section{Existence of solution for the coercive case}

In this section, we intend to prove the existence of solution for (1.1)-(1.2), by assuming that $a$ is coercive. Here, our main result has the following statement

Theorem 5.1. Assume that $\left(V_{1}\right)-\left(V_{4}\right)$ hold. If a is coercive, problem (1.1)-(1.2) has a solution $x \in H_{\text {loc }}^{1}(\mathbb{R}) \cap C^{2}(\mathbb{R})$ for all $\epsilon>0$. Moreover, $x(t) \in(0,1)$ for all $t \in \mathbb{R}$.

In the sequel, we will assume that $\epsilon=1$. However, in the proof of the above result, we must be careful to use the arguments of the previous sections, more precisely Sect. 2. In the sequel, we need to fix the following sets

$$
W_{a}=\left\{x \in H_{l o c}^{1}(\mathbb{R}): x+1 \in H_{a}^{1}((-\infty, 0]), x-1 \in H_{a}^{1}([0,+\infty))\right\}
$$

where

$$
H_{a}^{1}((-\infty, 0])=\left\{v \in H^{1}((-\infty, 0]): \int_{-\infty}^{0} a(t)|v(t)|^{2} d t<+\infty\right\}
$$

endowed with the norm

$$
\|v\|_{a,-\infty}=\left(\int_{-\infty}^{0}\left|v^{\prime}(t)\right|^{2} d t+\int_{-\infty}^{0} a(t)|v(t)|^{2} d t\right)^{\frac{1}{2}} .
$$

The space $H_{a}^{1}([0,+\infty))$ is defined of a similar way, that is,

$$
H_{a}^{1}([0,+\infty))=\left\{v \in H^{1}([0,+\infty)): \int_{0}^{+\infty} a(t)|v(t)|^{2} d t<+\infty\right\},
$$

endowed with the norm

$$
\|v\|_{a,+\infty}=\left(\int_{0}^{+\infty}\left|v^{\prime}(t)\right|^{2} d t+\int_{0}^{+\infty} a(t)|v(t)|^{2} d t\right)^{\frac{1}{2}} .
$$

From $\left(a_{10}\right)$, we know that $\inf _{t \in \mathbb{R}} a(t)>0$, then the embeddings

$$
H_{a}^{1}([0,+\infty)) \hookrightarrow H^{1}([0,+\infty)) \quad \text { and } \quad H_{a}^{1}((-\infty, 0]) \hookrightarrow H^{1}((-\infty, 0])
$$

are continuous. 


\section{Proof Theorem 5.1}

Hereafter, we follow the same approach of the previous section. Adapting the same arguments explored in [3], we can find sequences $\left(U_{n}\right) \subset W_{a}$, $\left(s_{n}\right),\left(t_{n}\right) \subset \mathbb{R}$ with $s_{n}<t_{n}$ satisfying:

$$
\begin{gathered}
J\left(U_{n}\right) \rightarrow \mathcal{B}, J^{\prime}\left(U_{n}\right) \rightarrow 0 \text { as } n \rightarrow+\infty, \\
U_{n}(t) \in[-1-1 / n,-1+\gamma+1 / n] \forall t \in\left(-\infty, s_{n}\right], \\
U_{n}(t) \in[1-\gamma-1 / n, 1+1 / n] \forall t \in\left[t_{n},+\infty\right), \\
U_{n}(t) \in[-1+\gamma-1 / n, 1-\gamma+1 / n] \forall t \in\left[s_{n}, t_{n}\right], \\
U_{n}\left(t_{n}\right)=1-\gamma+o_{n}(1), U_{n}\left(s_{n}\right)=-1+\gamma+o_{n}(1),
\end{gathered}
$$

and

$$
\left(t_{n}-s_{n}\right) \text { is bounded in } \mathbb{R} \text {. }
$$

A direct computation shows that for some subsequence of $\left(U_{n}\right)$, still denoted by itself, there is $U \in C(\mathbb{R}) \cap H_{l o c}^{1}(\mathbb{R})$ such that

$$
U_{n} \rightarrow U \text { uniformly in }[-T, T] \text { and } U_{n} \rightarrow U \text { in } H^{1}([-T, T]) \forall T>0 .
$$

Moreover,

$$
J(U) \leq \mathcal{B} \text { and } J^{\prime}(U)=0 .
$$

Here, $J$ and $\mathcal{B}$ are as in the proof of Theorem 4.1. Our goal is proving that $\mathcal{B}$ is achieved on $W$. To this end, we will study again the behavior of sequence $\left(s_{n}\right)$.

Claim 5.1. The sequence $\left(s_{n}\right)$ is bounded.

Arguing by contradiction, we will assume that $\left(s_{n}\right)$ is unbounded. Then for some subsequence, still denoted by itself, we have that

$$
s_{n} \rightarrow+\infty \text { or } s_{n} \rightarrow-\infty .
$$

Using the definition of $J$ and the properties of $\left(U_{n}\right)$, we derive that

$$
J\left(U_{n}\right) \geq V_{0} A_{n}\left(t_{n}-s_{n}\right),
$$

where

$$
A_{n}=\min _{z \in\left[s_{n}, t_{n}\right]} a(z) \text { and } V_{0}=\min _{-1+\gamma / 2 \leq z \leq 1-\gamma / 2} V(z)>0 .
$$

Using the fact that $a$ is coercive in conjunction with (5.2) and the boundedness of $\left(t_{n}-s_{n}\right)$, we deduce that

$$
A_{n} \rightarrow+\infty \text { as } n \rightarrow+\infty \text {. }
$$

Since $\left(J\left(U_{n}\right)\right)$ is bounded, the last inequality implies that

$$
t_{n}-s_{n} \rightarrow 0 \quad \text { as } n \rightarrow+\infty \text {. }
$$


On the other hand, we know that $\left(U_{n}\right) \subset H_{\text {loc }}^{1}(\mathbb{R})$, then for all $s, t \in \mathbb{R}$ the inequality below occurs

$$
\left|U_{n}(t)-U_{n}(s)\right| \leq \sqrt{|t-s|}\left(\int_{\min \{t, s\}}^{\max \{t, s\}}\left|U_{n}^{\prime}(r)\right|^{2} d r\right)^{\frac{1}{2}} \quad \forall n \in \mathbb{N} .
$$

Thus,

$$
\left|U_{n}(t)-U_{n}(s)\right| \leq \sqrt{2|t-s|} J\left(U_{n}\right)^{\frac{1}{2}} \quad \forall n \in \mathbb{N} .
$$

Now, the boundedness of $\left(J\left(U_{n}\right)\right)$ together with (5.3) gives

$$
\left|U_{n}\left(t_{n}\right)-U_{n}\left(s_{n}\right)\right| \rightarrow 0 \quad n \rightarrow+\infty .
$$

However, this limit cannot occur, because

$$
\left|U_{n}\left(t_{n}\right)-U_{n}\left(s_{n}\right)\right|=2-2 \gamma+o_{n}(1) \forall n \in \mathbb{N} .
$$

Therefore, the Claim 5.1 is proved.

Now, the proof of Theorem 5.1 follows the same steps of the proof of Theorem 4.1 .

\section{Final remarks}

In Sect. 2, we can remove the condition that $a \in L^{\infty}(\mathbb{R})$. Hovewer, we must work with the same spaces used in Sect. 5 .

\section{Acknowledgements}

The author is grateful to the referees for a number of helpful comments for improvement in this article.

\section{References}

[1] Alessio, F., Montecchiari, P.: Entire solutions in $\mathbb{R}^{2}$ for a class of Allen-Cahn equations. ESAIM: COCV 11, 633-672 (2005)

[2] Brézis, H.: Analyse Fonctionelle. Masson, Paris (1983)

[3] Bonheure, D., Sanchez, L.: Heteroclinic orbits for some classes of second and fourth order differential equation. In: Canãda, A., Drabek, P., Fonda, A. (eds.) Handbook of Differential Equations, Chapter 2. Elsevier, Amsterdam

[4] Bonheure, D., Obersnel, F., Omari, P.: Heteroclinic solutions of the prescribed curvature equation with a double-well potential. Differ. Integr. Equ. 26, 1411$1428(2013)$

[5] Bonheure, D., Sanchez, L., Tarallo, M., Terracini, S.: Heteroclinc connections between nonconsecutive equilibria of a fourth order differential equation. Calc. Var. Partial Differ. Equ. 17, 341-356 (2001) 
[6] Coti Zelati, V., Rabinowitz, P.H.: Heteroclinc solutions between stationay poinmts at different energy levels. Topol. Methods Nonlinear Anal. 17, 1-21 (2001)

[7] Ekeland, I.: On the variational principle. J. Math. Anal. Appl. 47, 324-353 (1974)

[8] Gavioli, A.: On the existence of heteroclinic trajectories for asymptotically autonomous equations. Topol. Method Nonlinear Anal. 34, 251-266 (2009)

[9] Gavioli, A.: Monotone heteroclinic solutions to non-autonomous equations via phase plane analysis. Nonlinear Differ. Equ. Appl. 18, 79-100 (2011)

[10] Gavioli, A., Sanchez, L.: Heteroclinic for non-autonomous second order differential equations. Differ. Integr. Equ. 22, 999-1018 (2009)

[11] Korman, P., Lazer, A.C., Li, Y.: On homoclinic and heteroclinic orbits for Hamiltonian systems. Differ. Integr. Equ. 10, 357-368 (1997)

[12] Spradlin, G.S.: Heteroclinic solutions to an asymptotically autonomous secondorder equation. EJDE 137, 1-14 (2010)

[13] Rabinowitz, P.H.: A new variational characterization of spatially heteroclinic solutions of a semilinear elliptic EDP. Discret. Contin. Dyn. Syst. 10, 507$515(2004)$

[14] Rabinowitz, P.H.: On a class of nonlinear Schrödinger equations. Z. Angew Math. Phys. 43, 270-291 (1992)

[15] Rabinowitz, P.H.: Homoclinic and heteroclinic orbits for a class of Hamiltonian systems. Calc. Var. Partial Differ. Equ. 1, 1-36 (1993)

Claudianor O. Alves

Universidade Federal de Campina Grande

Unidade Acadêmica de Matemática

Campina Grande, PB

CEP:58429-900

Brazil

e-mail: coalves@dme.ufcg.edu.br

Received: 20 September 2014.

Accepted: 28 February 2015. 